\title{
Learning curve in circular multipolar phased radiofrequency ablation of atrial fibrillation
}

\author{
Mihran Martirosyan, Alexandra Kiss, Edina Nagy-Baló, \\ Gábor Sándorfi, Diana Tint, István Édes, Zoltán Csanádi \\ Institute of Cardiology, University of Debrecen, Debrecen, Hungary
}

\begin{abstract}
Background: Although atrial fibrillation $(A F)$ ablation is considered a technically challenging procedure, studies on the learning curve of different pulmonary vein isolation (PVI) techniques are limited. We investigated the time-dependent changes in procedural parameters, complication rates, and in the 1-year clinical outcome during our initial experience with circular multipolar phased radiofrequency $(R F)$ ablation.

Methods and results: The first 132 consecutive patients (40 female; age: $56.6 \pm 10.4$ years) who underwent PVI with phased RF ablation for paroxysmal or persistent AF at our center were included in the study. Procedural parameters and atrial arrhythmia-free survival were compared in the first, second and third group of 44 successive patients. All pulmonary veins were successfully isolated in 44 (100\%), 41 (93.8\%) and 42 (95.5\%) patients in Tierce 1, 2 and 3 , respectively $(p=0.233)$. The number of $R F$ applications (per vein) required for isolation and fluoroscopy times demonstrated a significant decrease with experience, and a trend towards lower procedure times in Tierces 2 and 3 was also observed. Atrial arrhythmia-free survival rates at 12 months postablation were $68.18 \%, 75 \%$, and $70.75 \%$ in Tierce 1, Tierce 2 and Tierce 3, respectively ( $p=0.772)$. Pericardial tamponade requiring percutaneous subxiphoid drainage occurred in 1 patient (Tierce 3) as the only significant procedural complication. Conclusions: A learning curve effect was demonstrated in fluoroscopy times and in the number of RF applications but not in the acute success and in the long-term arrhythmia-free survival with circular multipolar RF ablations. (Cardiol J 2015; 22, 3: 260-266)
\end{abstract}

Key words: atrial fibrillation, phased radiofrequency ablation, learning curve, arrhythmia-free survival

\section{Introduction}

Catheter ablation for atrial fibrillation (AF) has emerged as an alternative to antiarrhythmic drug (AAD) therapy after the failure of at least one $\mathrm{AAD}$, or even as the first line of treatment in selected cases [1-3]. Although a wide variety of ablation techniques have been used to treat AF, it is generally agreed that the cornerstone of any transcatheter procedure is the electrical isolation of all pulmonary veins (PVs). This is currently most commonly achieved by encircling the PVs with focal radiofrequency $(\mathrm{RF})$ lesions under the guidance of a 3-dimensional electroanatomical mapping or navigation system. This point-by-point ablation technique requires extensive operator experience

Address for correspondence: Zoltan Csanadi MD, PhD, FESC, Institute of Cardiology, University of Debrecen, 22 Móricz Zs. Krt., Debrecen, Hungary, H-4032, tel: +36-209277231, fax: +3652414928, e-mail: drcsanadi@hotmail.com 
for efficiency and safety, and is usually associated with long procedure times. Novel methods aiming at simpler and faster PV isolation (PVI) have therefore been developed in recent years, including cryoballoon ablation (CBA) $[4,5]$ and multipolar RF ablation with the circular PV ablation catheter (PVAC) [6-9]. These "single-shot" techniques were designed to create a circular ablation lesion around the PVs after the appropriate positioning of the ablation catheter at the ostium or at the antra of each PV. A number of studies have demonstrated comparable levels of success and safety profile, but shorter procedure times with these simplified methods than with the conventional point-by-point ablation [10-13].

With the continuous increase in the number of $\mathrm{AF}$ ablation worldwide, the procedure is being introduced into new and less experienced centers. Efficacy and periprocedural complications are known to improve with experience in every invasive procedure including AF ablation although the learning curve effect has not been extensively evaluated. Sairaku et al. [14] have reported a significantly higher incidence of procedure-related complications and a lower arrhythmia-free survival at 6-month follow-up with double Lasso catheterguided encircling PVI in the first 52 as compared with the following 156 patients who underwent the ablation. Wójcik et al. [15] have demonstrated a learning curve effect with CBA as indicated by the decline in procedure and fluoroscopy times. However, improvement in the long-term outcome over time has been attributed to a more careful patient selection and not to operator experience per se.

We have now investigated the time-dependent changes in procedural parameters, complication rates, and in the 1-year clinical outcome during our initial experience with phased $\mathrm{RF}$ ablation.

\section{Methods}

\section{Study population}

The present study included consecutive patients who underwent PVI with phased RF ablation for paroxysmal or persistent AF at our center between November 01, 2009 and April 30, 2012, and who had regular follow-up during the first 12 months post-ablation. Exclusion criteria included previous AF ablation, long-standing persistent AF, hyper- and hypothyroidism, significant valvular heart disease, heart failure of New York Heart Association class III or IV, a left ventricular ejection fraction $\leq 40 \%$, a left atrial (LA) diameter exceeding $50 \mathrm{~mm}$, a LA thrombus, unstable angina or myocardial infarction within the last 3 months, severe chronic obstructive pulmonary disease, known bleeding disorders, contraindication to oral anticoagulation, and pregnancy. All participating patients signed the informed consent form prior to the procedure.

\section{Patient preparation and pre-procedural evaluation}

Patients were admitted to the hospital 1 or 2 days prior to the procedure. Those on oral anticoagulation with a vitamin $\mathrm{K}$ antagonist (VKA) continued to take the drug and the procedure was performed with an international normalized ratio in the therapeutic range. For all other patients, low molecular weight heparin was admnistered twice daily in a weight-adjusted dose and until $12 \mathrm{~h}$ prior to the procedure. All patients scheduled for ablation were examined by transesophageal echocardiography within $24 \mathrm{~h}$ to rule out an intracardiac thrombus. The LA and PV anatomies were assessed by means of multislice cardiac computed tomography imaging before the ablation.

\section{Ablation procedure with phased RF and the PVAC}

Ablation procedures were performed under conscious sedation with midazolam and fentanyl. Decapolar (BARD Electrophysiology Inc., Lowell, MA, USA) and quadripolar (Woxx $4 \mathrm{~J}, 6 \mathrm{~F}$, Biotronik, SE \& Co. KG, Berlin, Germany) catheters were advanced from the femoral vein and positioned into the coronary sinus and the right ventricle. Surface electrocardiograms (ECG) and bipolar intracardiac electrograms were registered with a Prucka, GE Medical digital recording system. A single transseptal puncture was performed under fluoroscopic guidance, by a standard technique using a Swartz SL (St. Jude Medical, Minneapolis, Mn, USA) transseptal sheath. This sheath was then exchanged for a deflectable 12 Fr CryoCath sheath (Medtronic CryoCath LP, Kirkland, Quebec, Canada) to be used for guiding in LA under continuous flush with heparinized saline. Immediately after the transseptal puncture, a $150 \mathrm{IU} / \mathrm{kg}$ body weight intravenous (i.v.) heparin bolus was administered, followed by a continuous infusion to maintain a minimum activated clotting time (ACT) target level of $>300$ s during ablations. Additional i.v. boluses of 2,000-5,000 IU heparin were administered as needed to attain the minimum target ACT level. This heparinization scheme was the same regardless of the ongoing rhythm (sinus rhythm vs. AF) during ablation.

The technical specifications of the circular multipolar PVAC, the GENius RF generator 
(Medtronic Inc., Minneapolis, MN, USA) and the methodology used in our center have been described in detail $[16,17]$. Briefly, the catheter was advanced through the FlexCath sheath over a 0.032-inch guide wire (BARD Electrophysiology Inc., Lowell, MA, USA), which was positioned selectively in each PV. The electrical conduction properties of the PV were assessed on the basis of the signals recorded by the PVAC electrodes after placement inside the ostium. Before the first RF delivery, the positions of the electrodes relative to the PV ostium were always confirmed by means of selective contrast injection through the FlexCath sheath. Care was taken to always apply the RF outside the vein in the antral region, targeting potentials of high amplitude on as many electrodes as possible for each application. Common ostia were isolated by inserting the guide wire into the different side branches and ablating subsequent segments of the targeted veins. The PVAC was connected to the GENius RF generator, which is capable of delivering RF current in different bipolar/unipolar mode ratios to any or all of the 5 bipolar channels in a duty-cycled mode. The target temperature was $60^{\circ} \mathrm{C}$, measured separately for all bipoles. Bipolar/unipolar RF delivery was usually started at a ratio of 4:1 for each PV and changed to a bipolar/unipolar proportion of 2:1 for a deeper lesion when a sufficient reduction in local electrogram amplitude could not be achieved after multiple RF deliveries. RF energy was applied for $60 \mathrm{~s}$, usually 3-4 times per PV, until PVI was achieved. The PV conduction was reassessed after each RF application, the electrodes being advanced inside the ostium.

\section{Follow-up}

Patients were usually discharged within 2 days after the ablation. Following the procedure a VKA was continued for at least 3 months. Patients taking an AAD before the procedure continued the medication for 3 months post-ablation. It was then discontinued if the patient was free of an AF relapse. VKA was discontinued 3 months after the ablation only in patients with a $\mathrm{CHA}_{2} \mathrm{DS}_{2}$ -VASc (Congestive heart failure, Hypertension, Diabetes mellitus, age $>75$ years; prior stroke or transient ischemic attack, Vascular disease, Age 65-74, female gender) score of 1 or below, while those patients with a higher stroke risk were kept on oral anticoagulation regardless of the results of postablation arrhythmia monitoring. Follow-up visits were scheduled at 6 weeks, and 3, 6, 9 and 12 months post-procedure.
The 12-lead ECG was checked at each follow-up. Additionally, 24-h Holter monitoring was performed at least twice, and also transtelephonic monitoring 3 times for 14-21 days, during the first 6 months. Patients were asked to transmit their rhythm at least 2-3 times a day, and always in the event of any palpitation. During non-telemetry periods of follow-up, patients were encouraged to visit the nearest hospital or outpatient facility to document their rhythm on an ECG whenever they felt any abnormality of their heart beat. Arrhythmia recurrence was defined as any atrial arrhythmia lasting for $30 \mathrm{~s}$ or longer. The definition of long-term success was freedom from any atrial arrhythmia without any AAD, after 1 procedure with a blanking period in the first 3 months.

\section{End points}

The acute endpoint of the procedure was the electrical isolation of all PVs, as confirmed by an entrance block.

Long-term efficacy was defined as freedom from any atrial arrhythmia without a Class I or Class III AAD after 1 procedure at 12 -month follow-up with a blanking period in the first 3 months.

Significant periprocedural complications were defined as any injury which resulted in death or had long-term sequel, requiring an immediate intervention or prolonged hospital stay.

\section{Statistical analysis}

The study period was divided into tierces to include the same number of patients who underwent $\mathrm{AF}$ ablation within each tierce. Clinical characteristics, procedural and follow-up data for subjects in each tierce were presented using numbers and frequencies (\%) for categorical variables and means with standard deviations for continuous variables. Statistical calculations were performed with IBM SPSS Statistics 20 Software.

The distribution was examined with Kolmogorov-Smirnov test. Discrete variables were analyzed using $\chi^{2}$ test. ANOVA (analysis of variances) and Kruskal-Wallis were used for comparisons of groups. Cox regression as univariate test was used to estimate the hazard ratio. $P$ value less than 0.05 was considered significant.

\section{Results}

A total of 132 patients were enrolled. Preablation clinical characteristics of the first, second and third group of 44 patients who underwent PVI with phased RF ablation are displayed in Table 1. 
Table 1. Baseline clinical characteristics in each tierce.

\begin{tabular}{lcccc}
\hline Patient characteristics & Tierce 1 & Tierce 2 & Tierce 3 & P \\
\hline Age & $55.12 \pm 10.13$ & $54.85 \pm 10$ & $59.82 \pm 10.45$ & 0.04 \\
Male/female & $33 / 11$ & $31 / 13$ & $28 / 16$ & 0.506 \\
Type of atrial fibrillation: persistent & $10(22.72 \%)$ & $9(20.45 \%)$ & $3(6.82 \%)$ & 0.096 \\
Medical history: & & & \\
$\quad$ Hypertension & $31(70.45 \%)$ & $29(65.9 \%)$ & $36(81.8 \%)$ & 0.225 \\
Diabetes & $5(11.36 \%)$ & $8(18.18 \%)$ & $7(15.9 \%)$ & 0.662 \\
Coronary artery disease & $4(9.1 \%)$ & $6(13.63 \%)$ & $9(20.45 \%)$ & 0.311 \\
Left atrial diameter [mm] & $40.61 \pm 4.6$ & $43.16 \pm 4.98$ & $42.11 \pm 4.35$ & 0.039 \\
Left ventricular ejection fraction [\%] & $55.45 \pm 6.1$ & $54.52 \pm 7.69$ & $55.43 \pm 7.95$ & 0.792 \\
\hline
\end{tabular}

Significant differences between the three tierces were found in the age and in the LA size only.

A total of $177 \mathrm{PVs}$ were successfully isolated out of the 177 targeted in Tierce 1, while 173/176 and $169 / 171$ in Tierces 2 and 3, respectively $(\mathrm{p}>0.05)$.

All PVs were successfully isolated in $44(100 \%)$, $41(93.8 \%)$ and $42(95.5 \%)$ patients in Tierce 1 , 2 and 3 , respectively $(\mathrm{p}=0.233)$. However, the number of RF applications (per PV) needed for isolation demonstrated a significant decrease with experience $(6.22 \pm 2.43 ; 4.65 \pm 1.32$ and $4.12 \pm$ \pm 1.2 in Tierce 1,2 and 3 , respectively; $p<0.001$ ). Procedure times demonstrated a trend towards lower values in Tierces 2 and 3 but the difference did not reach the level of statistical significance. In contrast, a significant decrease in fluoroscopy times was demonstrated (Fig. 1).

Pericardial tamponade requiring percutaneous subxiphoid drainage occurred in the $104^{\text {th }}$ consecutive patient (Tierce 3 ) as the only significant procedural complication.

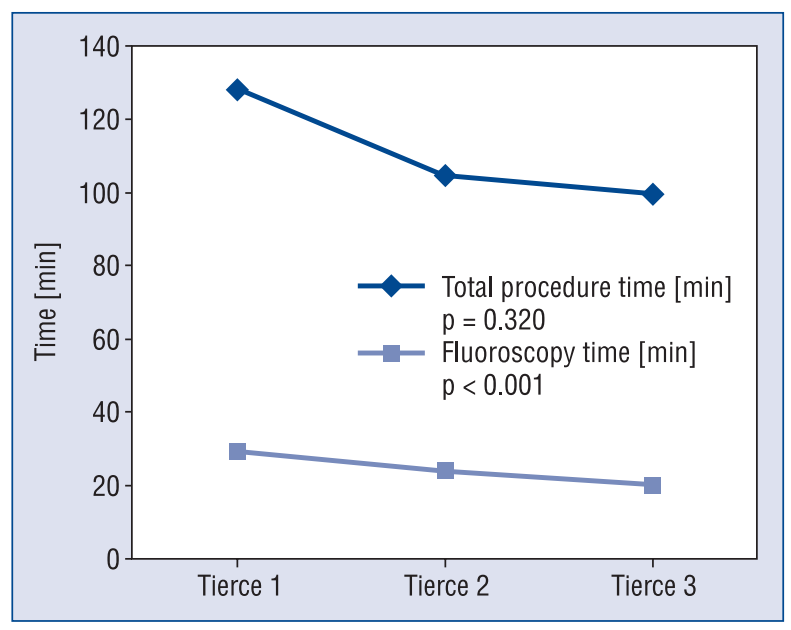

Figure 1. Procedure and fluoroscopy times in each tierce.
Atrial arrhythmia-free survival rates without $\mathrm{AAD}$ at 12 months postablation were $68 \%, 75 \%$, and $70.75 \%$ in Tierce 1 , Tierce 2 and Tierce 3, respectively $(\mathrm{p}=0.772)$. In Cox proportional hazard analysis which included clinical and procedural variables no significant predictor of arrhythmia recurrence was demonstrated (Table 2).

\section{Discussion}

\section{Main findings}

This study assessed the effects of learning curve on procedural parameters and long-term success during phased RF ablation with the PVAC. Fluoroscopy time and the number of RF applications required for successful PVI declined progressively with more experience, and a similar trend was observed for procedure time. Importantly, no learning curve effect was demonstrated in the success and complication rates.

\section{Previous studies on learning curve in AF ablation}

Although AF ablation is considered technically more challenging compared with other ablation procedures, very limited data have been published on the significance of operator experience in relation to the safety and efficacy of the procedure. The first study that specifically addressed the importance of the learning curve during AF ablation was published by Sairaku [14] who reported on the results of the first 208 consecutive PVIs with point-by-point focal RF ablation in a mediumvolume center. A significant learning curve effect has not only been demonstrated in procedure and fluoroscopy times, but also in complication rates and in the arrhythmia-free survival at 6 -month follow-up. In another study summarizing the experience, on $641 \mathrm{AF}$ ablations at Johns Hopkins 
Table 2. Cox regression analysis on 12-month arrhythmia-free survival.

\begin{tabular}{lccc}
\hline Variable & Hazard ratio & $\begin{array}{c}\text { AF free survival } \\
\text { 95\% confidence interval }\end{array}$ & P \\
\hline Gender & 1.352 & $0.589-3.106$ & 0.477 \\
Age & 1.019 & $0.978-1.061$ & 0.376 \\
Persistent AF & 1.291 & $0.542-3.078$ & 0.564 \\
Hypertension & 0.970 & $0.390-2.409$ & 0.947 \\
Coronary artery disease & 1.266 & $0.467-3.437$ & 0.643 \\
Diabetes & 0.808 & $0.301-2.165$ & 0.671 \\
Left atrial diameter & 1.001 & $0.925-1.083$ & 0.985 \\
Left ventricular ejection fraction & 0.988 & $0.940-1.038$ & 0.629 \\
Total procedure time & 0.996 & $0.987-1.005$ & 0.372 \\
Fluoroscopy time & 1.026 & $0.986-1.068$ & 0.207 \\
Group I & & & 0.839 \\
Group II & 0.777 & $0.337-1.793$ & 0.555 \\
Group III & 0.898 & $0.383-2.106$ & 0.805 \\
\hline
\end{tabular}

AF - atrial fibrillation

Hospital complication rates were $9 \%$ during the first 100 and $4.3 \%$ during the subsequent 541 procedures [18]. A worldwide survey [19] and the most recent consensus statement on $\mathrm{AF}$ ablation [1] also suggest that safety and efficacy results are better in centers performing more than 100 procedures annually. These recommendations are largely based on the experience gained with focal RF ablation.

Single-shot AF ablation techniques have been introduced to simplify and speed up PVI procedures. Available data on the the influence of operator experience as related to the safety and efficacy results with these simplified approaches are also limited. Wójcik et al. [15] reported on the procedural experience gained over 8 years with CBA in a high-volume center. A continuous decrease in fluoroscopy and procedure times was observed in each subsequent year and in multivariate analysis both the year of procedure and the preablation ALARMEc (Atrial fibrillation type, LA size, Renal insufficiency, MEtabolic syndrome, cardiomyopathy) risk score were independent predictors of procedure and fluoroscopy times. However, no significant decrease in complication rate over the 8 -year period has been demonstrated. The overall success rate at 12 months postablation was $73 \%$, which improved with each subsequent year, however this was related to the gradual fall in the ALARMEc risk score. In another single center study, the learning curves for PVI with phased RF ablation vs. with the cardiofocus laser balloon have been compared in the first 50 patients undergoing
PVI with each technology [20]. Procedure and fluoroscopy times decreased with time in both technologies. Atrial arrhythmia recurrence 6 months after a single procedure improved significantly from the first tierce $(31.2 \%)$ to the second $(17.6 \%)$ and to the third $(0 \%)$ with cardiofocus laser balloon but no clear improvement was found with phased $\mathrm{RF}$ ablation.

These published data are in line with our results and suggest that the influence of operator experience on clinical success and procedural complications of $\mathrm{AF}$ ablation may be less significant with a single-shot as compared with the conventional method. This is further supported by the initial experience of a center with AF ablation [21]. The first 109 patients at this center underwent PVI with either 3-dimensional guided focal RF or with phased $\mathrm{RF}$ ablation. The 6 -month success rate was significantly higher with phased $\mathrm{RF}(68 \%)$ as compared with focal (39\%) ablation, while complication rates were similar. Procedure and fluoroscopy times were also significantly shorter with phased RF ablation.

Although studies on direct comparison of PVAC with point-by-point PVI are limited, similar success rates have recently been reported with the two techniques [22]. In a multicenter prospective randomized comparison, the arrhythmia-free survival at 12 months was $56 \%$ with wide-area circumferential ablation and $60 \%$ with phased $\mathrm{RF}$ ablation in patients with paroxysmal AF. The efficacy of PVI with the PVAC without any other 
LA ablation target in more chronic forms of AF is yet to be determined [23]. Although the TTOP-AF study evaluated phased RF ablation in such patient cohort, other ablation catheters (multi-array ablation catheter and multi-array septal catheter) to target low amplitude high frequency complex fractionated electrograms on the LA posterior wall and septum were also used in that study. The ongoing Victory-AF trial is currently enrolling patients with persistent and long-standing persistent AF using PVAC GOLD the new generation of the PVAC catheter [24].

\section{Potential implications for clinical practice}

Available data suggest that the learning curve in PVI and the influence of previous operator experience on relevant procedural and clinical endpoints might be ablation technology-dependent. Ablation with a single-shot device can be performed not only with shorter procedure and fluoroscopy times as compared with focal ablation, but also with more satisfactory clinical outcome by a well-trained electrophysiologist who is proficient in transseptal catheterization and LA ablation but is in the early phase of his AF ablation practice. Further, any of these simplified approaches might be a reasonable choice for lower volume centers as a regular performance of a higher number of procedures is required not only to develop but also to maintain the adequate technical skills with point-by-point ablation.

\section{Limitations of the study}

This study has several limitations. First, this was a single center, observational patient cohort study including a relatively small number of patients thereby limiting the statistical power. There was, however, no selection bias for the study as consecutive patients undergoing AF ablation with phased RF were enrolled. Second, the main operator in all procedures had gained previous, although limited experience with CBA before he started this study, which required somewhat similar skills as ablation with the PVAC, therefore these results may not necessarily apply to what could be achieved by someone with absolutely no experience in AF ablation. Third, although high incidence of new silent cerebral ischemia detected by diffusion-weighted magnetic resonance imaging was reported after phased RF ablations [25, 26], this subclinical complication was not assessed in this study. However, most recent data indicated a very substantial reduction in silent cerebral embolization thanks to some technical and procedural modifications in phased RF ablation [27, 28].

\section{Conclusions}

This study investigated the time-dependent changes in procedural parameters, complication rates, and in the 1-year clinical outcome during our initial experience with circular, multipolar phased $\mathrm{RF}$ ablation. A learning curve effect was demonstrated in fluoroscopy times and in the number of $\mathrm{RF}$ applications but not in the acute success and in the long-term arrhythmia-free survival.

Conflict of interest: Zoltán Csanádi — speakers honoraria from Medtronic.

\section{References}

1. Calkins H, Kuck KH, Cappato R et al. 2012 HRS/EHRA/ECAS Expert Consensus Statement on Catheter and Surgical Ablation of Atrial Fibrillation: Recommendations for patient selection, procedural techniques, patient management and follow-up, definitions, endpoints, and research trial design. Europace, 2012; 14: 528-606.

2. Wilber DJ, Pappone C, Neuzil P et al. Comparison of antiarrhythmic drug therapy and radiofrequency catheter ablation in patients with paroxysmal atrial fibrillation: A randomized controlled trial. JAMA, 2010; 303: 333-340.

3. Camm JA, Lip GYH, De Caterina R et al. 2012 focused update of the ESC Guidelines for the management of atrial fibrillation. Eur Heart J, 2012; 33: 2719-2747.

4. Van Belle Y, Janse P, Rivero-Ayerza MJ et al. Pulmonary vein isolation using an occluding cryoballoon for circumferential ablation: Feasibility, complications, and short-term outcome. Eur Heart J, 2007; 28: 2231-2237.

5. Neumann T, Vogt J, Schumacher B et al. Circumferential pulmonary vein isolation with the cryoballoon technique results from a prospective 3-center study. J Am Coll Cardiol, 2008; 52: 273-278.

6. Boersma LV, Wijfels MC, Oral H, Wever EF, Morady F. Pulmonary vein isolation by duty-cycled bipolar and unipolar radiofrequency energy with a multielectrode ablation catheter. Heart Rhythm, 2008; 5:1635-1642.

7. Scharf C, Boersma L, Davies W et al. Ablation of persistent atrial fibrillation using multielectrode catheters and duty-cycled radiofrequency energy. J Cardiovasc Electrophysiol, 2010; 21: 399-405.

8. Boersama L, Duytschaever M, Geller JC, Scarf C. The OVAC Workbook. Remedica, London, UK 2010; 21: 41-67.

9. Wieczorek M, Hoeltgen R, Akin E, Salili AR, Oral H, Morady F. Results of short-term and long-term pulmonary vein isolation for paroxysmal atrial fibrillation using duty-cycled bipolar and unipolar radiofrequency energy. J Cardiovasc Electrophysiol, 2010; 2: 399-405.

10. Andrade JG, Khairy P, Guerra PG et al. Efficacy and safety of cryoballoon ablation for atrial fibrillation: A systematic review of published studies. Heart Rhythm, 2011; 8: 1444-1451.

11. Bulava A, Haniš J, Sitek D et al. Catheter ablation for paroxysmal atrial fibrillation: A randomized comparison between multielectrode catheter and point-by-point ablation. Pacing Clin Electrophysiol, 2010; 33: 1039-1046.

12. Richter B, Gwechenberger M, Kriegisch M, Albinni S, Marx M, Gössinger HD. Combined circular multielectrode catheter and point-by-point ablation is superior to point-by-point alone in eliminating atrial fibrillation. Int J Cardiol, 2013; 168: 3721-3727. 
13. Scharf C, Ng GA, Wieczorek M et al. European survey on efficacy and safety of duty-cycled radiofrequency ablation for atrial fibrillation. Europace, 2012; 14: 1700-1707.

14. Sairaku A, Nakano Y, Oda N, et al. Learning curve for ablation of atrial fibrillation in medium-volume centers. J Cardiol, 2011; 57: 263-268.

15. Wójcik M, Berkowitsch A, Greis $\mathrm{H}$ et al. Learning curve in cryoballon ablation of atrial fibrillation: Eight-year experience. Circulation, 2014; 78: 1612-1618.

16. Nagy-Baló E, Tint D, Clemens $\mathrm{M}$ et al. Transcranial measurement of cerebral microembolic signals during pulmonary vein isolation: A comparison of two ablation techniques. Circ Arrhythm Electrophysiol, 2013; 6: 473-480.

17. Kiss A, Nagy-Baló E, Hajas O, Édes I, Csanadi Z. Phased radiofrequency ablation for atrial fibrillation recurrence after cryoballoon ablation. Exp Clin Cardiol, 2014; 20: 2438-2443.

18. Spragg DD, Dalal D, Cheema A et al. Complications of catheter ablation for atrial fibrillation: Incidence and predictors. J Cardiovasc Electrophysiol, 2008; 19: 627-631.

19. Cappato R, Calkins H, Chen SA et al. Updated worldwide survey on the methods, efficacy, and safety of catheter ablation for human atrial fibrillation. Circ Arrhythm Electrophysiol, 2010; 3: 32-38.

20. Nguyen DQ, Lichtenberg L, Schuettler K, Fehske W. Retrospective comparison of the learning curves for PVI between Cardiofocus laser balloon and PVAC. JAFIB, 2013; October (special issue).

21. Choo WK, Farwell D, Harris S. Experience of atrial fibrillation ablation in a new cardiac centre using three-dimensional mapping and multielectrode duty-cycled radiofrequency ablation. Arch Cardiovasc Dis, 2011; 104: 396-402.

22. McCready J, Chow AW, Lowe MD et al. Safety and efficacy of multipolar pulmonary vein ablation catheter vs. irrigated radiofrequency ablation for paroxysmal atrial fibrillation: A randomized multicentre trial. Europace, 2014; 16: 1145-1153.

23. Hummel J, Michaud G, Hoyt $R$ et al. Phased RF ablation in persistent atrial fibrillation. Heart Rhythm, 2014; 11: 202-209.

24. ClinicalTrials.gov identifier: NCT01693120.

25. Gaita F, Caponi D, Pianelli M et al. Radiofrequency catheter ablation of atrial fibrillation: A cause of silent thromboembolism? Magnetic resonance imaging assessment of cerebral thromboembolism in patients undergoing ablation of atrial fibrillation. Circulation, 2010; 122: 1667-1673.

26. Deneke T, Shin DI, Balta O et al. Postablation asymptomatic cerebral lesions: Long-term follow-up using magnetic resonance imaging. Heart Rhtyhm, 2011; 8: 1705-1711.

27. Verma A, Debruyne P, Nardi S et al. Evaluation and reduction of asymptomatic cerebral embolism in ablation of atrial fibrillation, but high prevalence of chronic silent infarction: Results of the evaluation of reduction of asymptomatic cerebral embolism trial. Circ Arrhythm Electrophysiol, 2013; 6: 835-842.

28. Kiss A, Nagy-Baló E, Sandorfi G, Édes I, Csanadi Z. Cerebral microembolization during atrial fibrillation ablation: Comparison of different single-shot ablation techniques. Int J Cardiol, 2014; 174: 276-281. 